\title{
Dynamics from a mathematical model of a two-state gas laser
}

\author{
Antigoni Kleanthous, Tianshu Hua, Alexandre Manai, Kamran Yawar, and Robert A. Van Gorder* \\ Mathematical Institute, University of Oxford, Andrew Wiles Building, Radcliffe Observatory Quarter, Woodstock Road, Oxford, OX2 6GG, UK \\ *Robert.VanGorder@maths.ox.ac.uk
}

\begin{abstract}
Motivated by recent work in the area, we consider the behavior of solutions to a nonlinear PDE model of a two-state gas laser. We first review the derivation of the two-state gas laser model, before deriving a non-dimensional model given in terms of coupled nonlinear partial differential equations. We then classify the steady states of this system, in order to determine the possible long-time asymptotic solutions to this model, as well as corresponding stability results, showing that the only uniform steady state (the zero motion state) is unstable, while a linear profile in space is stable. We then provide numerical simulations for the full unsteady model. We show for a wide variety of initial conditions that the solutions tend toward the stable linear steady state profiles. We also consider traveling wave solutions, and determine the unique wave speed (in terms of the other model parameters) which allows wave-like solutions to exist. Despite some similarities between the model and the inviscid Burger's equation, the solutions we obtain are much more regular than the solutions to the inviscid Burger's equation, with no evidence of shock formation or loss of regularity.
\end{abstract}

Keywords: two-state gas laser; nonlinear dynamics; stability analysis; numerical simulations

\section{Introduction}

A gas laser is a laser which is usually activated by passing a sufficiently large current (either continuous, or pulsed) through the gas. Other ways to activate the gas laser are also possible (chemical pumping, and optical pumping) but are not as common [1]. Gas lasers consist of different states of atoms which become excited upon activation. Once a given species is in its excited state, it can decay to lower states, including the ground state, by four different processes: (a) collisions between an electron and the excited species, (b) near-resonant collisions between the excited species and the same or a different species in the ground state, (c) collisions with the walls of the container, and (d) spontaneous emission [1]. One common example of a two-state gas laser would be a helium-neon laser [2, 3, 4].

Dynamics akin to turbulence have been observed experimentally in specific gas laser systems [5]. In order to simulate all of the processes at play, one would require a fairly complicated mathematical model. Therefore, in order to better understand the qualitative features of turbulence in gas lasers, simpler caricature models, which preserve the important qualitative features of the gas laser system while neglecting complicated and perhaps unnecessary terms, are desirable. A recent study by Solon et al. [6] considered an interesting mathematical model of a two-state gas laser system. In this work, the authors suggested that turbulent behaviour exists in this two-state gas laser. In particular, they obtained an approximate solution which had multiple branches, and concluded that this multi-branch structure would induce turbulence in the full system. However, the approach of Solon et al. [6] for deducing turbulence had a flaw, as they did not actually establish that the solutions were multi-valued. Rather, they made some approximations, and then concluded that the approximation to the solutions was multi-valued, without checking that the approximation was a true representation of the actual solution. As it turns out, they did not solve the needed system simultaneously, and therefore their approximation is not valid. As the analysis was all based on this approximate solution, their conclusions about turbulence are not really substantiated.

Despite the fact that no exact or accurate numerical solution was considered in [6], the model discussed in [6] is physically interesting, and should be considered further. There have been various analytical studies on mathematical equations arising in the study of turbulence [7, 8,9], yet, to the best of our knowledge, no analytical results on gas laser dynamics under models such as that proposed in [6] exist in the literature. Motivated by this, in the present paper we 
perform a more detailed mathematical and numerical analysis of this model, in order to better understand the behavior of solutions to this two-state system. In Section 2 we discuss a derivation of the two-state gas laser model. In Section 3 , we non-dimensionalise and obtain a mathematical formulation in terms of coupled nonlinear partial differential equations. In Section 4, we find the uniform and non-uniform steady states for this model, and deduce their stability. In Section 5, we turn our attention to the full unsteady model, and provide numerical simulations. For a wide variety of initial conditions, we show that the solutions tend toward the stable steady state profiles, which are linear functions of the spatial variable. In Section 6 we focus on traveling wave solutions, and again these tend to behave like linear functions. Despite some similarities between the equations, the solutions we obtain are much more regular than the solutions to the inviscid Burger's equation, and in Section 7 we discuss why this is true. We give concluding remarks in Section 8.

\section{Mathematical Model for a Two-State Gas Laser}

We begin with a review of the gas laser model of [6]. We consider a volume of gas molecules which consists of two different types of particles; the excited and the ground state. Take $\mathbf{v}$ to be the velocity of the gas at any space coordinate $\mathbf{x}$ and time $t$. Then, let $f_{1}(\mathbf{x}, \mathbf{v}, t)$ be the density distribution function for the ground state particles and $f_{2}(\mathbf{x}, \mathbf{v}, t)$ the density distribution function for the excited state particles. The evolution of $f_{1}$ and $f_{2}$ is then given by the Boltzmann's Transport Equation. If we define $\mathbf{f}(\mathbf{x}, \mathbf{v}, t)=\left(f_{1}(\mathbf{x}, \mathbf{v}, t), f_{2}(\mathbf{x}, \mathbf{v}, t)\right)^{T}$, then the distribution evolves like

$$
\mathbf{f}(\mathbf{x}+\mathbf{v} d t, \mathbf{v}, t+d t)=\mathbf{f}(\mathbf{x}, \mathbf{v}, t) .
$$

The quantities $\mathbf{v} d t$ and $d t$ are very small. Considering a Taylor series expansion

$$
\mathbf{f}(\mathbf{x}+\mathbf{v} d t, \mathbf{v}, t+d t)=\mathbf{f}(\mathbf{x}, \mathbf{v}, t)+d t\left(\frac{\partial \mathbf{f}}{\partial t}+\mathbf{v} \cdot \nabla_{x} \mathbf{f}\right)+O\left(d t^{2}\right)
$$

which upon using Equation (1) and considering terms of $O(d t)$ becomes

$$
\frac{\partial \mathbf{f}}{\partial t}=-\mathbf{v} \cdot \nabla_{\mathbf{x}} \mathbf{f}
$$

Now, if a collision occurs, we need to add a term that explains the effect of the collision of the time evolution of $\mathbf{f}$, that is

$$
\frac{\partial \mathbf{f}}{\partial t}=-\mathbf{v} \cdot \nabla_{\mathbf{x}} \mathbf{f}+\left(\frac{\partial \mathbf{f}}{\partial t}\right)_{\text {collision }} .
$$

We assume that only binary collisions occur as the probability of 3 or more particles to collide is very small. We further assume that the velocity and position of a molecule are uncorrelated (molecular chaos assumption [10]). If we only consider collision terms due to transmission caused by interaction with a radiation bath, according to [6] we can take

$$
\left(\frac{\partial \mathbf{f}}{\partial t}\right)_{\text {collision }}=\left(\begin{array}{l}
A f_{2}+\rho B_{21} f_{2}-\rho B_{12} f_{1} \\
A f_{1}-\rho B_{21} f_{2}+\rho B_{12} f_{1}
\end{array}\right) .
$$

Here $A$ and $B$ are the Einstein coefficients [11]. The coefficient $A$ represents the rate of spontaneous emission, $\rho$ is the density of the radiation field, $\rho B_{21}$ is the stimulated rate of emission, and $\rho B_{12}$ is the rate of stimulated absorption.

We consider the motion along the axis of propagation of the laser, and therefore take $\mathbf{x}=x$. Substituting Equation (5) into (4) we obtain in components of $\mathbf{f}$

$$
\begin{aligned}
& \frac{\partial f_{1}}{\partial t}=-v \frac{\partial f_{1}}{\partial x}+A f_{2}+\rho B_{21} f_{2}-\rho B_{12} f_{1}, \\
& \frac{\partial f_{2}}{\partial t}=-v \frac{\partial f_{2}}{\partial x}+A f_{1}-\rho B_{21} f_{2}+\rho B_{12} f_{1} .
\end{aligned}
$$


The above equations, describe the time evolution of the density field for the gas particles. It is more reasonable to consider the velocity of the densities of particles, taking the view that the ground and excited states overall behave like fluids. Consider the transformations [6]

$$
v_{i}=\int f_{i}(x, v, t) v(x, t) d v
$$

where $v_{i}=v_{i}(x, t)$ for $i=1,2$. Multiplying Equations (6) and (7) by $v$, and then integrating over velocity space, we obtain the system in [6]

$$
\begin{aligned}
\frac{\partial v_{1}}{\partial t} & =-\frac{1}{2} \frac{\partial}{\partial x}\left(v_{1}^{2}\right)+A v_{2}+\rho B_{21} v_{2}-\rho B_{12} v_{1} \\
\frac{\partial v_{2}}{\partial t} & =-\frac{1}{2} \frac{\partial}{\partial x}\left(v_{2}^{2}\right)+A v_{1}-\rho B_{21} v_{2}+\rho B_{12} v_{1}
\end{aligned}
$$

Note that the above, is a non-linear, coupled system of PDEs. Furthermore, if $v_{2} \equiv 0, A=B_{21}=B_{12}=0$, we have inviscid Burger's Equation

$$
\frac{\partial v_{1}}{\partial t}+\frac{1}{2} \frac{\partial}{\partial x}\left(v_{1}^{2}\right)=0
$$

Equations such as (11) are known to have shocks [12]. Hence we will study Equations (9)-(10) to see if any complicated dynamics are possible, such as the turbulent behaviour as suggested in [6].

In this generalized model, a photon is emitted on de-excitation in a random direction. Regarding coherent radiation, there will still be emission of light from the underlying laser apparatus under the dynamics we consider. However, in our model, we focus on the gas reservoir of the gas laser. One important feature which may change, due to turbulence or other non-equilibrium dynamics in the laser, is the efficiency of the laser. Of course, the properties of the light emitted by the laser will depend on many other aspects of the laser apparatus.

In order to activate the laser, we need to take the steady state from rest $v_{1}=v_{2}=0$, to some non zero steady state. One approach is to change the initial state, $V_{1}(x), V_{2}(x)$ so that at least one is non zero. This is an intrinsic change, as we are selecting the initial velocity field completely. The other approach is to act on a system through some external action, say an applied force acted at some time $t=T$. This force could continue to be applied for $t>T$, or could be instantaneous.

It may make more sense to apply the external action to the ground state $v_{1}$, since these gas molecules may be more plentiful. The system with external force would then take the form

$$
\begin{aligned}
& \frac{\partial v_{1}}{\partial t}+\frac{1}{2} \frac{\partial}{\partial x}\left(v_{1}^{2}\right)=-\rho B_{12} v_{1}+\left(A+\rho B_{21}\right) v_{2}+\phi_{1}(x, t), \\
& \frac{\partial v_{2}}{\partial t}+\frac{1}{2} \frac{\partial}{\partial x}\left(v_{2}^{2}\right)=\left(A+\rho B_{12}\right) v_{1}-\rho B_{21} v_{2}+\phi_{2}(x, t),
\end{aligned}
$$

where $\phi_{i}$ is the forcing function. The forcing function $\phi_{1}$ will modify the velocity profile of the ground state, while the forcing function $\phi_{2}$ will modify the velocity profile of the excited state. Different functions for $\phi_{i}$ are considered later for the numerical simulation.

\section{Non-Dimensional Mathematical Model}

We assume that the tube of the gas laser has finite length $L$. The full mathematical problem with initial and periodic boundary conditions is then given by Equations (12)-(13) with initial data

$$
v_{1}(x, 0)=V_{1}(x), v_{2}(x, 0)=V_{2}(x),
$$

and appropriate periodic boundary data, where $V_{1}$ and $V_{2}$ are specified functions. Note that if $V_{1}(x) \equiv 0$ and $V_{2}(x) \equiv 0$ then $v_{1}(x, t)=v_{2}(x, t)=0$ is always a solution. 
As mentioned in [6], in physical units

$$
B_{12}=\frac{\pi}{3 \epsilon_{0} \hbar^{2}} p_{12}, B_{21}=\frac{\pi}{3 \epsilon_{0} \hbar^{2}} p_{21}, A=\frac{\omega^{3} \hbar}{c^{2}} B
$$

where $p_{i j}>0$ is the probability of transition from state $i$ to $j, \omega$ is the angular frequency, $\hbar$ is Planck's constant, $c$ is the speed of light, $\epsilon_{0}$ is the permittivity of free space and $B=B_{12}=B_{21}$ (although $B_{12} \neq B_{21}$ in general). Note that the energy between the ground and excited state is $\tilde{E}=\hbar \omega$.

For the purpose of this paper we consider a more general case $B_{12} \neq B_{21}$, where

$$
B=\frac{1}{2}\left(B_{12}+B_{21}\right), A=\frac{\omega^{3} \hbar}{2 c^{2}}\left(B_{12}+B_{21}\right) .
$$

Note that this choice is not unique, and different values may be taken. We rescale the system so that units correspond to $c=\epsilon_{0}=\hbar=1$. Then the coefficients become

$$
B_{12}=\frac{\pi}{3} p_{12}, B_{21}=\frac{\pi}{3} p_{21}, A=\frac{\tilde{E}^{3}}{2}\left(p_{12}+p_{21}\right)
$$

Using the following quantities

$$
\hat{t}=\frac{\rho \pi}{3} t, \hat{x}=\frac{\rho \pi}{3} x, E^{3}=\frac{3}{2 \rho \pi} \tilde{E}^{3}
$$

and defining $v_{i}(x, t)=\hat{v}_{i}(\hat{x}, \hat{t})$ and $\phi_{i}(x, t)=\frac{3}{\rho \pi} \hat{\Phi}_{i}(\hat{x}, \hat{t})$ in the new coordinates we obtain the non-dimensional system

$$
\begin{aligned}
& \frac{\partial \hat{v_{1}}}{\partial \hat{t}}+\frac{1}{2} \frac{\partial}{\partial \hat{x}}\left(\hat{v}_{1}^{2}\right)=-p_{12} \hat{v_{1}}+\left[E^{3}\left(p_{12}+p_{21}\right)+p_{21}\right] \hat{v_{2}}+\hat{\Phi}_{1}(x, t), \\
& \frac{\partial \hat{v_{2}}}{\partial \hat{t}}+\frac{1}{2} \frac{\partial}{\partial \hat{x}}\left(\hat{v}_{2}^{2}\right)=\left[E^{3}\left(p_{12}+p_{21}\right)+p_{12}\right] \hat{v_{1}}-p_{21} \hat{v_{2}}+\hat{\Phi}_{2}(x, t) .
\end{aligned}
$$

Defining

$$
\mu_{12}=E^{3}\left(p_{12}+p_{21}\right)+p_{12}, \quad \mu_{21}=E^{3}\left(p_{12}+p_{21}\right)+p_{21},
$$

and dropping the hats, we obtain the full non-dimensional system

$$
\begin{gathered}
\frac{\partial v_{1}}{\partial t}+\frac{1}{2} \frac{\partial}{\partial x}\left(v_{1}^{2}\right)=-p_{12} v_{1}+\mu_{21} v_{2}+\Phi_{1}(x, t), \\
\frac{\partial v_{2}}{\partial t}+\frac{1}{2} \frac{\partial}{\partial x}\left(v_{2}^{2}\right)=\mu_{12} v_{1}-p_{21} v_{2}+\Phi_{2}(x, t), \\
v_{1}(x, 0)=V_{1}(x) \quad, \quad v_{2}(x, 0)=V_{2}(x),
\end{gathered}
$$

with appropriate boundary data. Note that $\mu_{12}>0$ and $\mu_{21}>0$, since $E, p_{12}$, and $p_{21}$ are positive. We now have a system involving three parameters, each of which represents properties of the gas laser: $E=O(1)$ is the energy required to go from the ground state to the first excited state, $p_{12} \in[0,1]$ is the probability of stimulated absorption, and $p_{21} \in[0,1]$ is the probability of stimulated emission.

\section{Stability Analysis for Steady State Solutions}

We now consider stability of solutions which are initially small, and which are not acted on by external forces, i.e. $\Phi_{1}=\Phi_{2}=0$. In general, we let $\tilde{v}_{1}, \tilde{v}_{2}$ be steady state solutions independent of time. We may consider small perturbations about these solutions, say

$$
\begin{aligned}
& v_{1}(x, t)=\tilde{v}_{1}(x)+\delta w_{1}(x, t)+O\left(\delta^{2}\right), \\
& v_{2}(x, t)=\tilde{v}_{2}(x)+\delta w_{2}(x, t)+O\left(\delta^{2}\right),
\end{aligned}
$$


where $|\delta| \ll 1$ is a small perturbation parameter and $\left|w_{1}\right|,\left|w_{2}\right|=O(1)$. Substituting in the system we obtain the order $O(1)$ equations

$$
\begin{aligned}
& \tilde{v}_{1} \frac{d \tilde{v}_{1}}{d x}=-p_{12} \tilde{v}_{1}+\mu_{21} \tilde{v}_{2}, \\
& \tilde{v}_{2} \frac{d \tilde{v}_{2}}{d x}=\mu_{12} \tilde{v}_{1}-p_{21} \tilde{v}_{2},
\end{aligned}
$$

and the $O(\delta)$ equations

$$
\begin{aligned}
& \frac{\partial w_{1}}{\partial t}+\tilde{v}_{1} \frac{\partial w_{1}}{\partial x}+w_{1} \frac{d \tilde{v}_{1}}{d x}=-p_{12} w_{1}+\mu_{21} w_{2}, \\
& \frac{\partial w_{2}}{\partial t}+\tilde{v}_{2} \frac{\partial w_{2}}{\partial x}+w_{2} \frac{d \tilde{v}_{2}}{d x}=\mu_{12} w_{1}-p_{21} w_{2} .
\end{aligned}
$$

In order to determine stability of the steady states governed by (27)-(28), we must show that the perturbations governed by (29)-(30) remain small or decay to zero as $t \rightarrow+\infty$. On the other hand, if they grow, then the steady states will be unstable. Since the solution of the $O(\delta)$ terms depends on the solution to the $O(1)$ equations, we shall consider all relevant cases of the $O(1)$ solutions.

\subsection{Instability of the Uniform Zero Steady State}

Let $\tilde{v_{1}} \equiv \tilde{v_{2}} \equiv 0$. Note that this is the only spatially uniform steady state possible. Then, the $O(\delta)$ solutions give

$$
\begin{aligned}
& \frac{\partial w_{1}}{\partial t}=-p_{12} w_{1}+\mu_{21} w_{2} \\
& \frac{\partial w_{2}}{\partial t}=\mu_{12} w_{1}-p_{21} w_{2} .
\end{aligned}
$$

The Jacobian in this case is given by

$$
J=\left(\begin{array}{cc}
-p_{12} & \mu_{21} \\
\mu_{12} & -p_{21}
\end{array}\right)
$$

with corresponding eigenvalues

$$
\lambda_{1,2}=\frac{1}{2}\left(p_{12}+p_{21}\right)\left(-1 \pm \sqrt{1+4 E^{3}\left(E^{3}+1\right)}\right) .
$$

Note that $E>0$, which implies that the square root is always greater than one, giving two distinct real values. We therefore have one positive and one negative eigenvalue, which leads us to the conclusion that the steady state $\left(\tilde{v}_{1}, \tilde{v}_{2}\right)=(0,0)$ is unstable.

\subsection{Non-Existence of Non-Zero Uniform Steady States}

A positive uniform steady state will not always exist. We would need for there to be a positive solution to the system

$$
\begin{aligned}
-p_{12} \tilde{v}_{1}+\mu_{21} \tilde{v}_{2} & =0, \\
\mu_{12} \tilde{v}_{1}-p_{21} \tilde{v}_{2} & =0 .
\end{aligned}
$$

Hence,

$$
\begin{aligned}
& \tilde{v}_{2}=\frac{p_{12}}{\mu_{21}} \tilde{v}_{1}, \\
& \tilde{v}_{2}=\frac{\mu_{12}}{p_{21}} \tilde{v}_{1},
\end{aligned}
$$

which implies $p_{12} p_{21}=\mu_{12} \mu_{21}$, and by substituting the values of $\mu_{12}$ and $\mu_{21}$, we arrive at

$$
p_{12} p_{21}=E^{6}\left(p_{12}+p_{21}\right)^{2}+E^{3}\left(p_{12}+p_{21}\right)^{2}+p_{12} p_{21}
$$

and therefore $E=0$. So, a positive steady state can exist only if $E=0$, which is not physical. As such, we do not consider this case further. 


\subsection{Stability of a Non-Uniform Steady State}

To find a non-uniform steady state, we are required to first solve Equations (27)-(28) where we can write $\tilde{v}_{2}$ in terms of $\tilde{v}_{1}$

$$
\tilde{v}_{2}=\frac{p_{12}}{\mu_{21}} \tilde{v}_{1}+\frac{1}{\mu_{21}} \tilde{v}_{1} \frac{d \tilde{v}_{1}}{d x}
$$

Placing this in Equation (28), we obtain a single second order non-linear ODE

$$
\left(p_{12} \tilde{v}_{1}^{2}+\tilde{v}_{1}^{2} \frac{d \tilde{v}_{1}}{d x}\right) \frac{d^{2} \tilde{v}_{1}}{d x^{2}}+\tilde{v}_{1}\left(\frac{d \tilde{v}_{1}}{d x}\right)^{3}+2 p_{12} \tilde{v}_{1}\left(\frac{d \tilde{v}_{1}}{d x}\right)^{2}+\left(p_{12}^{2}+p_{21} \mu_{21}\right) \tilde{v_{1}} \frac{\tilde{v_{1}}}{d x}-\mu_{21}\left(\mu_{21} \mu_{12}-p_{12} p_{21}\right) \tilde{v_{1}}=0 .
$$

Although this is an ODE, it is still complicated and cannot be solved directly, but we can attempt to find some particular solutions.

Consider, for instance, $\tilde{v}_{1}=\beta x$, a linear function in space. Then the ODE reduces to a cubic equation for $\beta$ :

$$
q(\beta)=\beta^{3}+2 p_{12} \beta^{2}+\left(p_{12}^{2}+p_{21} \mu_{21}\right) \beta-\mu_{21}\left(\mu_{21} \mu_{12}-p_{12} p_{21}\right)=0 .
$$

We know that $\mu_{21}\left(\mu_{21} \mu_{12}-p_{12} p_{21}\right)>0$ for $E>0$, hence $q(0)<0$. Yet, $q \rightarrow \infty$ as $\beta \rightarrow \infty$. Therefore, there must exist at least one positive $\operatorname{root} \beta=\bar{\beta}>0$. For that root, $\tilde{v_{1}}=\bar{\beta} x$ is an exact solution to Equation (41). Then we can obtain $\tilde{v}_{2}$

$$
\tilde{v}_{2}(x)=\frac{\left(p_{12}+\bar{\beta}\right) \bar{\beta}}{\mu_{21}} x=\beta^{*} x
$$

which is also a linear function in space. In this way, we have found one particular non-uniform steady state. We can attempt to calculate $\bar{\beta}$ numerically, and for physically meaningful and fixed values of $p_{i j}$, $\mu_{i j}$, we find one positive solution.

We can now place the steady states $\tilde{v}_{1}$ and $\tilde{v}_{2}$ into the Equations (29)-(30) to obtain

$$
\begin{aligned}
\frac{\partial w_{1}}{\partial t}+\bar{\beta}\left(x \frac{\partial w_{1}}{\partial x}+w_{1}\right) & =-p_{12} w_{1}+\mu_{21} w_{2}, \\
\frac{\partial w_{2}}{\partial t}+\frac{\left(p_{12}+\bar{\beta}\right) \bar{\beta}}{\mu_{21}}\left(x \frac{\partial w_{2}}{\partial x}+w_{2}\right) & =\mu_{12} w_{1}-p_{21} w_{2} .
\end{aligned}
$$

From the form of these equations, we try $w_{1}(x, t)=\hat{\beta}_{1}(t) x$ and $w_{2}(x, t)=\hat{\beta}_{2}(t) x$. These are of the same form (linear in space) of the $O(1)$ solutions, yet can account for temporal changes. Substituting we find that

$$
\begin{aligned}
\frac{d \hat{\beta}_{1}}{d t}+2 \bar{\beta} \hat{\beta}_{1} & =-p_{12} \hat{\beta}_{1}+\mu_{21} \hat{\beta}_{2}, \\
\frac{d \hat{\beta}_{2}}{d t}+2 \frac{\left(p_{12}+\bar{\beta}\right) \bar{\beta}}{\mu_{21}} \hat{\beta}_{2} & =\mu_{12} \hat{\beta}_{1}-p_{21} \hat{\beta}_{2} .
\end{aligned}
$$

Note that $\left(\hat{\beta}_{1}, \hat{\beta}_{2}\right)=(0,0)$ is clearly a fixed point. If we show that this point is stable, then the solution is stable under perturbation. The Jacobian matrix in this case is given by

$$
J\left(\hat{\beta}_{1}, \hat{\beta}_{2}\right)=\left(\begin{array}{cc}
-p_{12}-2 \bar{\beta} & \mu_{21} \\
\mu_{12} & -p_{21}-2 \frac{\left(p_{12}+\bar{\beta}\right) \bar{\beta}}{\mu_{21}}
\end{array}\right) .
$$

If $\operatorname{tr}(J)<0$ and $\operatorname{det}(J)>0$, then we have two eigenvalues with negative real parts, and the linear stability for the system is guaranteed. In this case,

$$
\operatorname{tr}(J)=-\left(p_{12}+p_{21}+2 \bar{\beta}+2 \frac{\left(p_{12}+\bar{\beta}\right) \bar{\beta}}{\mu_{21}}\right),
$$



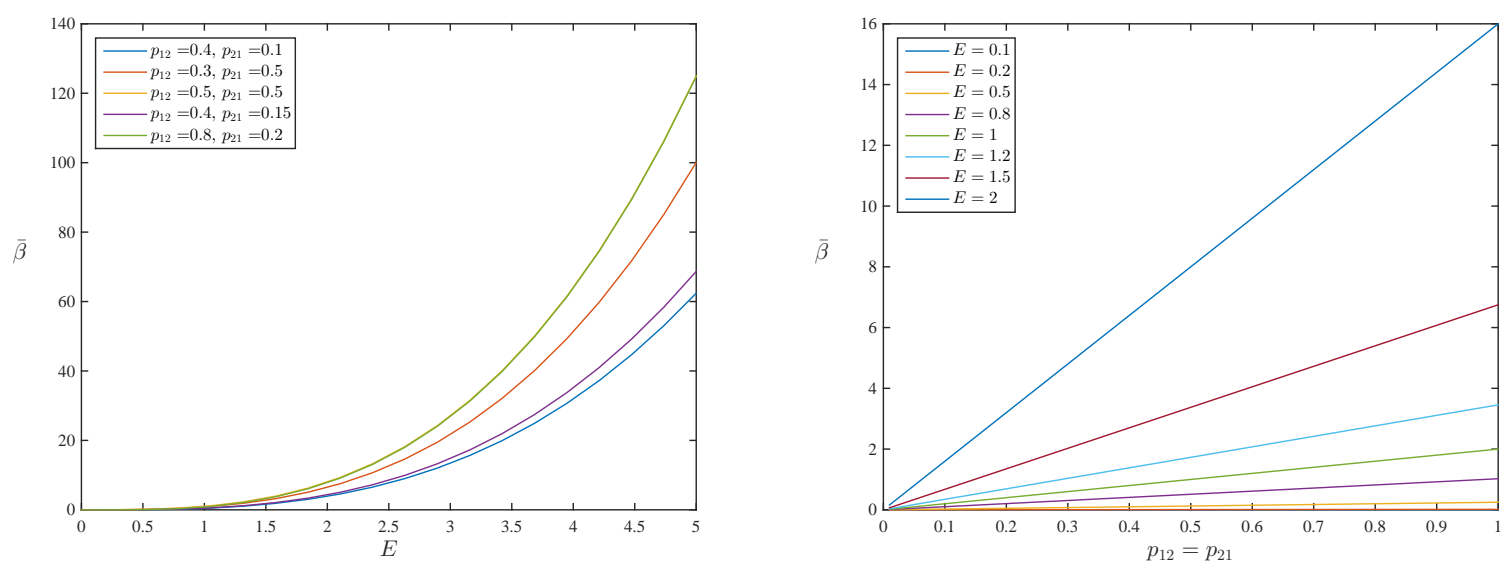

Figure 1: The slope $\bar{\beta}$ of the linear steady state plotted against $E$ for different pairs $p_{12}, p_{21}$ in the first plot and against $p_{12}=p_{21}$ for different values of energy $E$ in the second one.

which is always negative since each term inside the brackets is positive, and

$$
\operatorname{det}(J)=\left(p_{12}+2 \bar{\beta}\right)\left(p_{21}+2 \frac{\left(p_{12}+\bar{\beta}\right) \bar{\beta}}{\mu_{21}}\right)-\mu_{12} \mu_{21},
$$

which becomes

$$
\mu_{21} \operatorname{det}(J)=4 \bar{\beta}^{3}+6 p_{12} \bar{\beta}^{2}+2\left(p_{12}^{2}+p_{21} \mu_{21}\right) \bar{\beta}+\mu_{21}\left(p_{12} p_{21}-\mu_{12} \mu_{21}\right) .
$$

Recall that $\bar{\beta}$ satisfies Equation (42). Subtracting (42) from the above, we get

$$
\mu_{21} \operatorname{det}(J)=3 \bar{\beta}^{3}+4 p_{12} \bar{\beta}^{2}+\left(p_{12}^{2}+3 p_{21} \mu_{21}\right) \bar{\beta} .
$$

Recall that we have assumed that $\bar{\beta}>0$. Therefore since all the coefficients on the right hand side are positive then we can conclude that $\operatorname{det}(J)>0$. This implies that the linear steady state $\left(\tilde{v}_{1}(x), \tilde{v}_{2}(x)\right)=\left(\bar{\beta} x, \beta^{*} x\right)$ is locally stable under perturbation.

To get a better understanding of how the slopes $\bar{\beta}$ and $\beta^{*}$ of the linear steady states behave for different values of $p_{12}, p_{21}$ and $E$, we plot the graphs using MATLAB as seen in Figure 1. The graphs for $\beta^{*}$ are omitted as they exhibit similar behaviour to $\bar{\beta}$, in fact they look almost identical. After further investigation we find that the difference between $\bar{\beta}$ and $\beta^{*}$ is near machine precision. The slopes are therefore approximately the same $\bar{\beta} \approx \beta^{*}$. Notice that although we plot 5 cases, we can only see 4 in the first plot. This is because the slopes are dominated by the behaviour of $p_{12}+p_{21}$ instead of $p_{12}$ and $p_{21}$ independently, and therefore the green one $\left(p_{12}=0.8, p_{21}=0.2\right)$ lies on top of the yellow one $\left(p_{12}=0.5, p_{21}=0.5\right)$. We observe that the slope $\bar{\beta}$ grows algebraically with increasing $E$. We then proceed to plot the slope $\bar{\beta}$ against $p_{12}=p_{21}$; again the graph for $\beta^{*}$ is omitted for similar reasons. We observe that for increasing values of $p_{12}=p_{21}$ the slope grows linearly for all values of $E$ examined, and that for greater values of $E$, we have a greater slope.

\subsection{On the Approximated Steady States of [6]}

In Section 1 we mentioned that the approximate solution of [6] for the steady states was not correct (due to the manner in which it was derived). We are finally at a point where we may demonstrate why this is.

Starting with (27)-(28), let us make the same assumption as [6] that $\tilde{v}_{2}=\tilde{v}_{20}$, a constant. Then, the system (27)-(28) is put into the form

$$
\begin{aligned}
\tilde{v}_{1} \frac{d \tilde{v}_{1}}{d x} & =-p_{12} \tilde{v}_{1}+\mu_{21} \tilde{v}_{2} \\
0 & =\mu_{12} \tilde{v}_{1}-p_{21} \tilde{v}_{20} .
\end{aligned}
$$


The latter equation implies that $\tilde{v}_{1}$ must also be a constant, $\tilde{v}_{1}=\tilde{v}_{10}$, and this means that the derivative term in the first equation vanishes, leaving us with a system of two linear algebraic equations for the two unknown $\tilde{v}_{10}$ and $\tilde{v}_{20}$. However, as mentioned above, there exist no positive uniform steady states, so the only possible solution is $\tilde{v}_{10}=0$ and $\tilde{v}_{20}=0$.

The error made in [6] is that the steady state system was not solved simultaneously, as is required. Rather, the authors only considered (53) after assuming $\tilde{v}_{2}=\tilde{v}_{20}$ to be a constant. They then solved the ODE (53), obtaining a spurious solution, which they then placed into (54) in order to find a solution for $\tilde{v}_{2}$. They considered this solution in their subsequent analysis. They did not stop to consider more terms in their approximation, or to place the approximations back into the original steady state equations in order to ascertain the accuracy of their solutions. The solution to (53) alone involves a constant term, a linear term, and a term involving a Lambert W function. However, had they noted that the only possibility for $\tilde{v}_{20}$ was $\tilde{v}_{20}=0$, then the Lambert W term would have vanished. Yet, it was the Lambert W term that provided their multi-branched solution. Hence, the claim of [6] that (27)-(28) has multi-valued solutions is seen to be incorrect. This highlights the need to actually check that an approximate solution is both derived correctly and does indeed accurately represent the true solution to the problem.

\section{Numerical Simulation of the Non-Dimensional Full Model}

We now proceed to investigate the behaviour of the velocity profiles $v_{1}$ and $v_{2}$ numerically. We discretize the space $\{(x, t): 0 \leq x \leq L, 0 \leq t \leq T\}$ with a spatial grid size $h$, a temporal step $\Delta t$ and the grid points $\left(x_{j}, t^{n}\right)$ as follows

$$
\begin{aligned}
x_{j} & =j h, j=1,2, \ldots, M, \\
t^{n} & =n \Delta t, n=1,2, \ldots, N .
\end{aligned}
$$

We discretize time using the Lax-Friedrichs Scheme [13]

$$
\frac{\partial v_{i}}{\partial t}(n \Delta t, j h)=\frac{1}{\Delta t}\left[v_{i_{j}}^{n+1}-\frac{1}{2}\left(v_{i_{j+1}}^{n}+v_{i_{j-1}}^{n}\right)\right], i=1,2,
$$

and discretize space using Forward Euler [13]

$$
\frac{\partial v_{i}}{\partial x}(n \Delta t, j h)=\frac{1}{h}\left[v_{i_{j+1}}^{n}-v_{i_{j}}^{n}\right], i=1,2 .
$$

We choose the Lax-Friedrichs Scheme as it is suitable for hyperbolic PDEs. We use MATLAB to implement the above algorithm.

We saw earlier that the system can be set into motion either by imposing some initial conditions or by acting some external forcing function $\Phi_{1}, \Phi_{2}$. We investigate both cases here.

\subsection{Influence of Initial Conditions on Solution Behaviour}

In this case, we set the forcing functions $\Phi_{1}=\Phi_{2}=0$ to investigate the behaviour of the system for different initial conditions as can be seen in Figures 2-5. In all cases we assume the laser has length $L=\pi$, zero boundary conditions $v_{1}(0, t)=v_{1}(L, t)=v_{2}(0, t)=v_{2}(L, t)=0$, probabilities of transition $p_{12}=0.4, p_{21}=0.6$, and energy $E=1$. Although boundary conditions weren't necessary for the mathematical analysis earlier, they are necessary for the numerical simulation.

The sharp variation in solutions for small time observed in Figures 2, 3 and 5 is because of the initial conditions for $v_{1}$ being nonzero at the boundaries, while the boundary conditions are set to zero. The non-smoothness at the beginning is smoothed out as time progresses. Note also, that the curves are required to return back to zero at the end of the laser due to the boundary conditions being zero, which explains the shape of the curve observed.

In all four cases, the velocity profiles $v_{1}$ and $v_{2}$ approach the linear steady state suggested by the mathematical analysis earlier (indicated with a bright dashed red line in Figures 2-5). Note that the initial conditions used in Figure 5 give shocks in the inviscid Burger's equation [12]. Therefore, solutions of (22)-(23) appear better behaved than their counterparts when $p_{12}=p_{21}=0$. It is important to note the uniqueness of the linear steady state. In Figure 3, although we set a linear state as an initial condition, the velocity $v_{1}$ diverges from that initial linear state to approach the linear steady state with slope $\bar{\beta}$ as was derived earlier in Equation (42). 

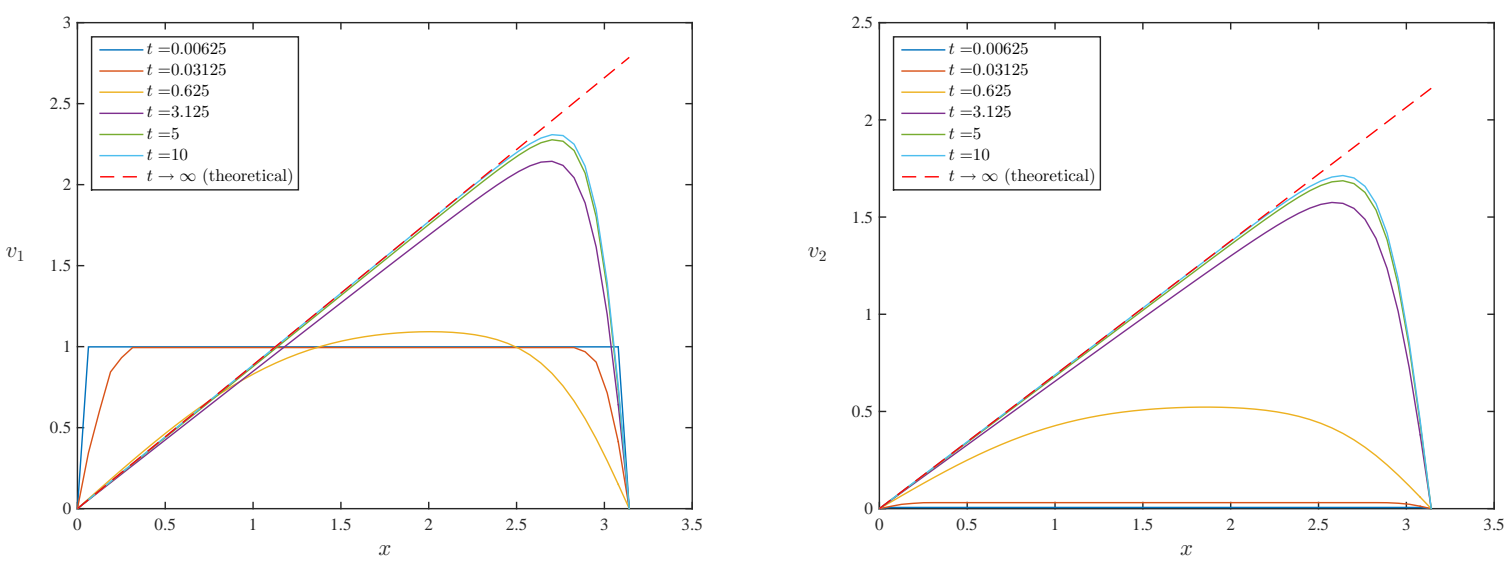

Figure 2: Velocity profiles $v_{1}, v_{2}$ against $x$ for different time values $t$, for initial conditions $v_{1}(x, 0)=1$ and $v_{2}(x, 0)=0$.
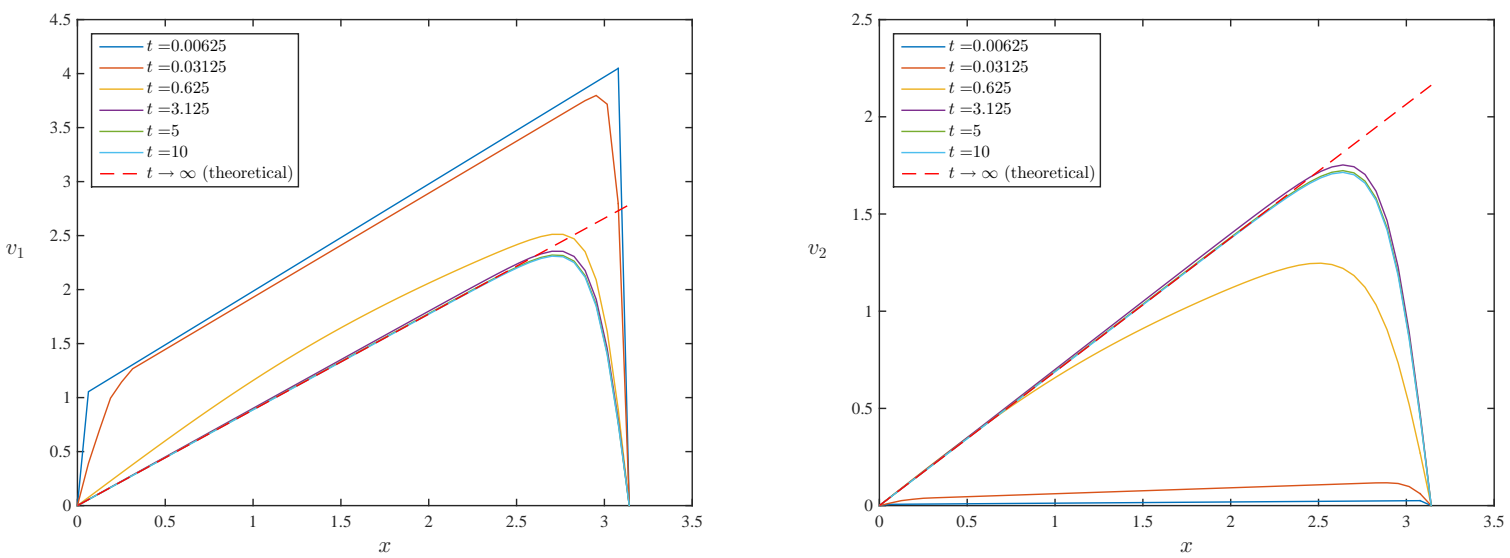

Figure 3: Velocity profiles $v_{1}, v_{2}$ against $x$ for different time values $t$, for initial conditions $v_{1}(x, 0)=x+1$ and $v_{2}(x, 0)=0$.
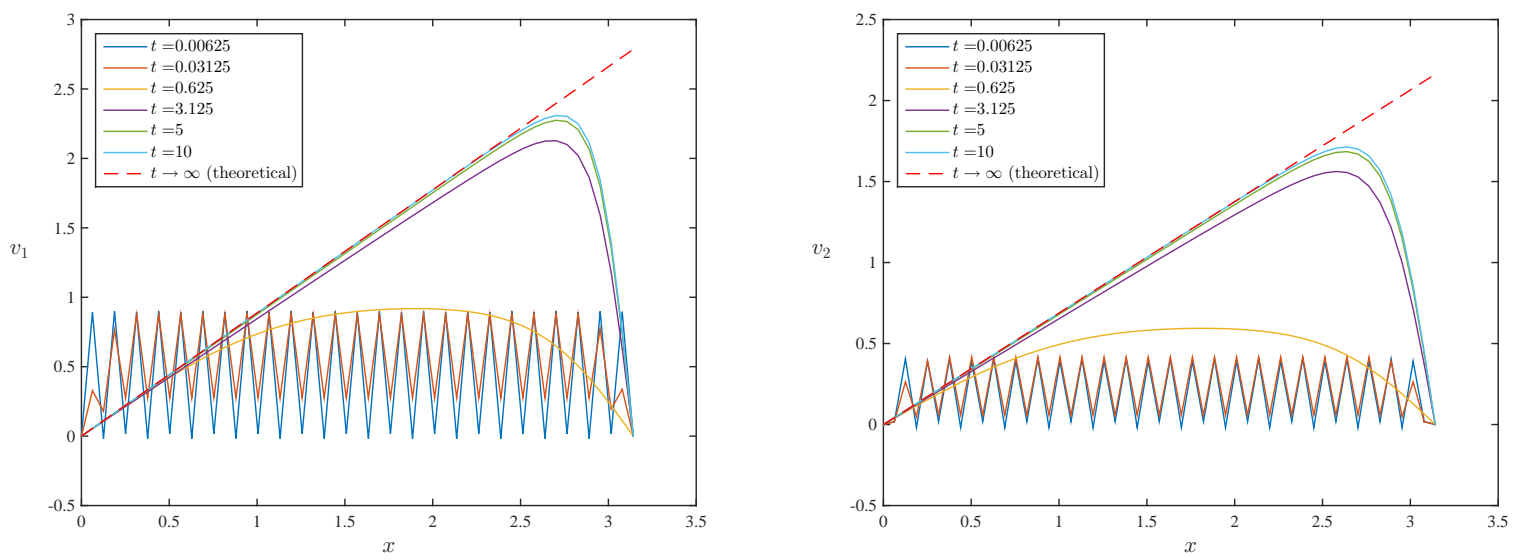

Figure 4: Velocity profiles $v_{1}, v_{2}$ against $x$ for different time values $t$, for initial conditions $v_{1}(x, 0)=3 \sin \left(\frac{25 \pi x}{L}\right)$ and $v_{2}(x, 0)=2 \cos \left(\frac{25 \pi x}{L}\right)$. 

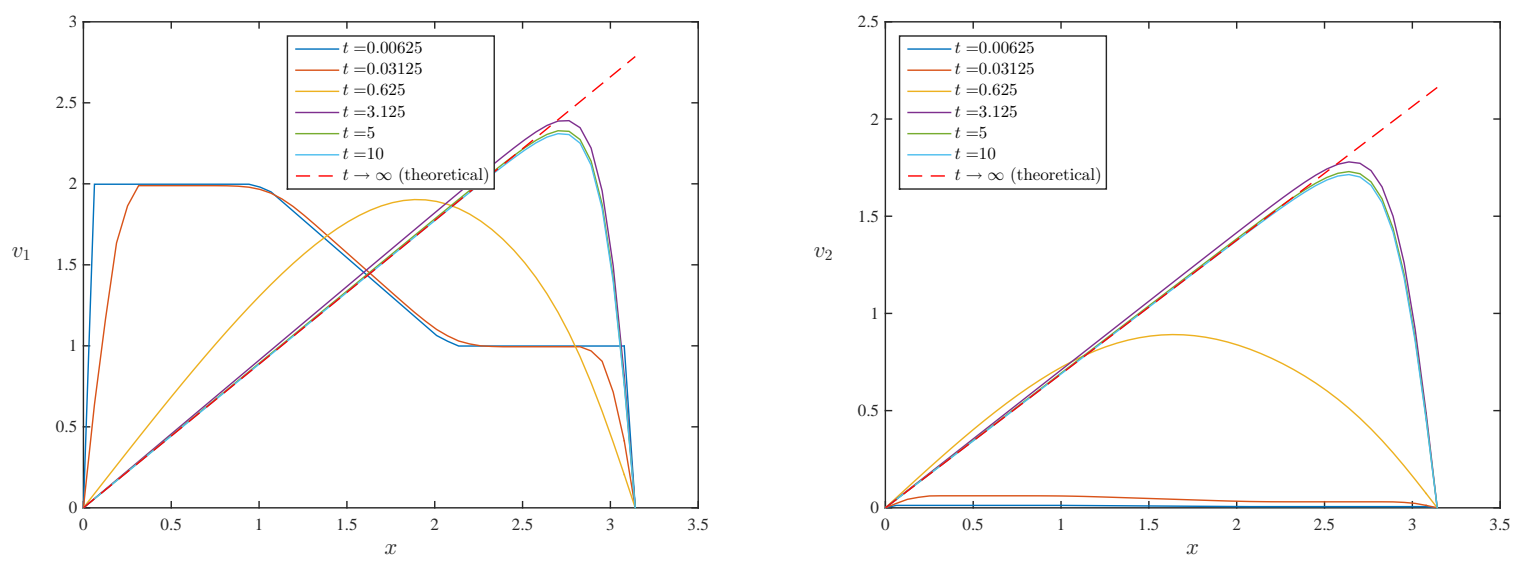

Figure 5: Velocity profiles $v_{1}, v_{2}$ against $x$ for different time values $t$, for initial conditions $v_{1}(x, 0)=2$ for $0 \leq x<\frac{\pi}{3}, v_{1}(x, 0)=3-3 x / \pi$ for $\frac{\pi}{3} \leq x<\frac{2 \pi}{3}, v_{1}(x, 0)=1$ for $\frac{2 \pi}{3} \leq x<\pi$ and $v_{2}(x, 0)=0$.
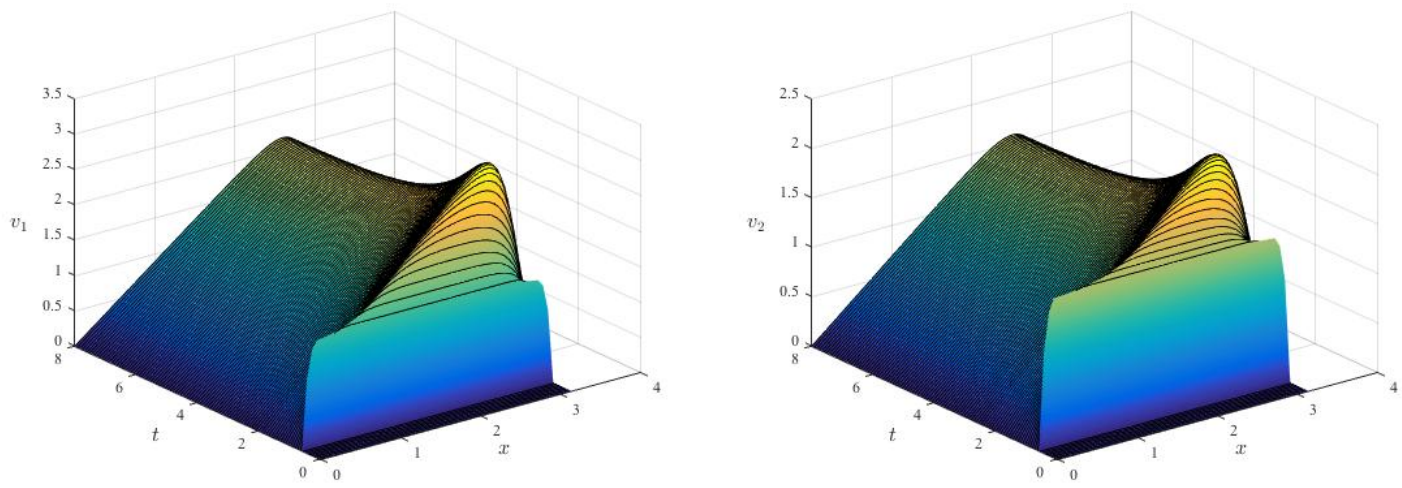

Figure 6: Velocity profiles $v_{1}, v_{2}$ for forcing function $\Phi(x, t)=1.5 \delta(t-T)$ for $T=60$

\subsection{Influence of Forcing Functions on Solution Behavior}

We now consider the case where $v_{1}(x, 0) \equiv 0, v_{2}(x, 0) \equiv 0$, and the system is driven by forcing functions $\Phi_{1}$, $\Phi_{2} \neq 0$. If we apply the same force to each species, then $\Phi_{1}=\Phi_{2}=\Phi$, for some forcing function $\Phi=\Phi(x, t)$. From the form of the PDE system, there are two types of forces; $\Phi>0$, in which case we have an amplifying force, which increases the time derivative of the velocity field, and $\Phi<0$, in which case we have a damping force, which decreases the derivative of the velocity field.

We consider several types of forcing functions. The results can be seen in Figures 6-8. In all cases we have zero initial conditions $v_{1}(x, 0)=v_{2}(x, 0,) \equiv 0$, zero boundary conditions $v_{1}(0, t)=v_{1}(L, t)=0, v_{2}(0, t)=v_{2}(L, t)=0$, probabilities of transition $p_{12}=0.4, p_{21}=0.6$, and energy $E=1$. We observe that no matter how the forcing function looks like, the velocity profiles of $v_{1}$ and $v_{2}$ appear to always approach a linear steady state which depends more strongly on the values of $E, p_{12}$, and $p_{21}$, rather than on the initial conditions or even the forcing term.

\subsection{Solutions for Various Non-Dimensional Lengths}

In an attempt to see how the length of the laser affects the velocity profile, we run the simulation for different non-dimensional lengths $L$ keeping any other parameters constant. In this case, we have initial conditions $v_{1}(x, 0)=$ $3 \sin (25 \pi x / L)$ and $v_{2}(x, 0)=2 \cos (25 \pi x / L)$, with zero boundary conditions $v_{1}(0, t)=v_{1}(L, t)=v_{2}(0, t)=v_{2}(L, t)=0$, zero forcing functions $\Phi_{1}=\Phi_{2} \equiv 0$, probabilities of transition $p_{12}=0.4, p_{21}=0.6$, and energy $E=1$. The results for 
$\Delta$ $-$ 
$v_{2}$ can be seen in Figure 9. Solutions for $v_{1}$ are omitted as they exhibit similar behaviour to that of $v_{2}$. In all three cases the velocity profiles approach the linear steady state as expected from the mathematical analysis. We observe that the maximum velocity increases for increased length but a slower convergence to the linear steady state is observed for greater lengths, requiring more time to approach the linear steady state.

\section{Traveling Wave Solution}

We now look at a specific solution type, a traveling wave solution with wave speed $c$, in order to see if there are any qualitative distinctions from the solutions found in previous sections. The assumption of traveling wave solutions is fairly common in the study of nonlinear evolution equations, as it permits one to reduce the underlying PDE(s) into ODE(s) which are often more tractable for analytical approaches; see, for instance $[14,15,16,17,18]$.

We let $\eta=x-c t$ be the new variable, such that $v_{1}(x, t)=u_{1}(\eta)$ and $v_{2}(x, t)=u_{2}(\eta)$, and end up with a system of ODEs

$$
\begin{aligned}
& \frac{d u_{1}}{d \eta}\left(u_{1}-c\right)=-p_{12} u_{1}+\mu_{21} u_{2}+\Phi_{1}(\eta), \\
& \frac{d u_{2}}{d \eta}\left(u_{2}-c\right)=\mu_{12} u_{1}-p_{21} u_{2}+\Phi_{2}(\eta) .
\end{aligned}
$$

We are taking $\Phi_{1}$ and $\Phi_{2}$ to be constant for the rest of the paper.

The steady states $\left(u_{1}^{*}, u_{2}^{*}\right)$ can be found by setting $d u_{1} / d t=d u_{2} / d t=0$

$$
\left(u_{1}^{*}, u_{2}^{*}\right)=\left(\frac{p_{12} \Phi_{1}+\mu_{21} \Phi_{2}}{p_{21} p_{12}-\mu_{12} \mu_{21}}, \frac{\mu_{12} \Phi_{1}+p_{12} \Phi_{2}}{p_{12} p_{21}-\mu_{12} \mu_{21}}\right) .
$$

In the case where there are no forcing functions, i.e. $\Phi_{1}=\Phi_{2}=0$, the steady state is $(0,0)$, with the Jacobian matrix given by

$$
J=\left(\begin{array}{cc}
\frac{p_{12}}{c} & -\frac{\mu_{21}}{c} \\
-\frac{\mu_{12}}{c} & \frac{p_{21}}{c}
\end{array}\right) .
$$

In this case

$$
\operatorname{tr}(J)=\frac{1}{c}\left(p_{12}+p_{21}\right)>0
$$

and

$$
\operatorname{det}(J)=\frac{1}{c^{2}}\left(p_{12} p_{21}-\mu_{12} \mu_{21}\right)=-\frac{1}{c^{2}}\left(p_{12}+p_{21}\right)^{2} E^{3}\left(1+E^{3}\right)<0 .
$$

This implies that one eigenvalue is negative while the other is positive, which means that $(0,0)$ is an unstable steady state. This agrees with the analysis of the PDE non-dimensional system.

\subsection{Linear Solution}

It is reasonable to try a linear solution $u_{1}(\eta)=\beta_{1} \eta+\alpha_{1}$ to see if the results agree with the original PDE system. Here $\alpha$ can be interpreted as the initial value of $u_{1}(0)$. Substituting into Equation (59) we obtain

$$
u_{2}=\beta_{2} \eta+\alpha_{2},
$$

where

$$
\begin{aligned}
& \beta_{2}=\frac{\beta_{1}\left(\beta_{1}+p_{12}\right)}{\mu_{21}} \\
& \alpha_{2}=\frac{\beta_{1}\left(\alpha_{1}-c\right)+p_{12} \alpha_{1}-\Phi_{1}}{\mu_{21}}
\end{aligned}
$$


and substituting into Equation (60) we get

$$
\beta_{2}\left(\beta_{2} \eta+\alpha_{2}\right)=\mu_{12}\left(\beta_{1} \eta+\alpha_{1}\right)-p_{21}\left(\beta_{2} \eta+\alpha_{2}\right)+\Phi_{2}
$$

Equating the coefficients of $O(\eta)$ and substituting the quantities $\beta_{2}$ and $\alpha_{2}$ we obtain

$$
q\left(\beta_{1}\right)=\beta_{1}^{3}+2 p_{12} \beta_{1}^{2}+\left(p_{12}^{2}+p_{21} \mu_{21}\right) \beta_{1}-\mu_{21}\left(\mu_{21} \mu_{12}-p_{12} p_{21}\right)=0,
$$

which is identical to Equation (42)! Following the same reasoning there exists a positive $\beta_{1}=\bar{\beta}>0$. Taking the coefficients of $O(1)$ we obtain a formula for the wave speed $c$,

$$
c=-\frac{\mu_{21}^{2} \Phi_{2}+\left[\beta\left(\beta+p_{12}\right)+\mu_{21} p_{21}\right] \Phi_{1}}{\beta\left(\beta+p_{12}\right)\left(\beta+\mu_{21}\right)+\beta p_{21} \mu_{21}} .
$$

Notice that the wave speed $c$ doesn't depend on the initial velocity $\alpha_{1}$, and hence if $\Phi_{1}=\Phi_{2}=0$ then $c=0$ and the traveling wave solution degenerates into a steady state solution. Therefore, in order to obtain a linear solution

$$
\begin{aligned}
& u_{1}(\eta)=\bar{\beta} \eta+\alpha_{1} \\
& u_{2}(\eta)=\frac{\bar{\beta}\left(\bar{\beta}+p_{12}\right)}{\mu_{21}} \eta+\frac{\bar{\beta}\left(\alpha_{1}-c\right)+p_{12} \alpha_{1}-\Phi_{1}}{\mu_{21}}
\end{aligned}
$$

we need non-zero forcing functions $\Phi_{1}$ and $\Phi_{2}$.

\subsection{Numerical Simulation}

To study the behaviour of $u_{1}$ and $u_{2}$ further, we proceed with the numerical simulation of (59)-(60) using MATLAB. We use a 4th order Runge-Kutta Scheme for the discretization of the system. As before we take the transition probabilities to be $p_{12}=0.4, p_{21}=0.6$ and energy $E=1$.

We run the software for different values of wave speed $c$ with fixed forcing functions $\Phi_{1}, \Phi_{2}$ in the first plot of Figure 10. We observe that increasing values of $c$, increase $u_{1}$. We omit the plot of $u_{2}$ again as it exhibits similar behaviour. The numerical simulation is only valid for values $c<\min \left\{u_{1}(0), u_{2}(0)\right\}$ due to the non-linearity of the left hand side of Equations (59)-(60) causing a discontinuity when $c$ approaches either $u_{1}(0)$ or $u_{2}(0)$. For any value $c \geq \min \left\{u_{1}(0), u_{2}(0)\right\}$ the numerical scheme breaks down. In the second plot of Figure 10 we plot $u_{1}$ for different values of $\Phi=\Phi_{1}=\Phi_{2}$ and the corresponding fixed values for $c, u_{1}(0)$ and $u_{2}(0)$. The starred red line corresponds to the theoretical linear solution for $u_{1}$. We can observe that it matches the numerical solution for the corresponding values. We also notice that positive values of $\Phi$ increase $u_{1}$ while negative values decrease them.

\section{Comparison with Inviscid Burger's Equation}

We observed a number of solutions, yet these all tended toward linear steady states in space, for large time. This implies that solutions to the gas laser model have great regularity, even though this system is essentially a generalization of the inviscid Burger's equation. In this section, we suggest a reason for this greater regularity.

Since many of the solutions at steady state were scalar multiples of the other, let us consider the case where

$$
v_{2}(x, t)=\sigma v_{1}(x, t)
$$

where $\sigma$ is a parameter to be determined. Taking this assumption in the non-dimensional system (22)-(23), we have that both equations are consistent only if

$$
1=\sigma=\frac{\mu_{12}+p_{12}}{\mu_{21}+p_{21}}=\frac{E^{3}\left(p_{12}+p_{21}\right)+2 p_{12}}{E^{3}\left(p_{12}+p_{21}\right)+2 p_{21}} .
$$

One case where this holds is $p_{12}=p_{21}$, so we shall consider this case. The system (22)-(23) is then equivalent to the single equation

$$
\frac{\partial v_{1}}{\partial t}+\frac{1}{2} \frac{\partial}{\partial x}\left(v_{1}^{2}\right)=\alpha v_{1}
$$



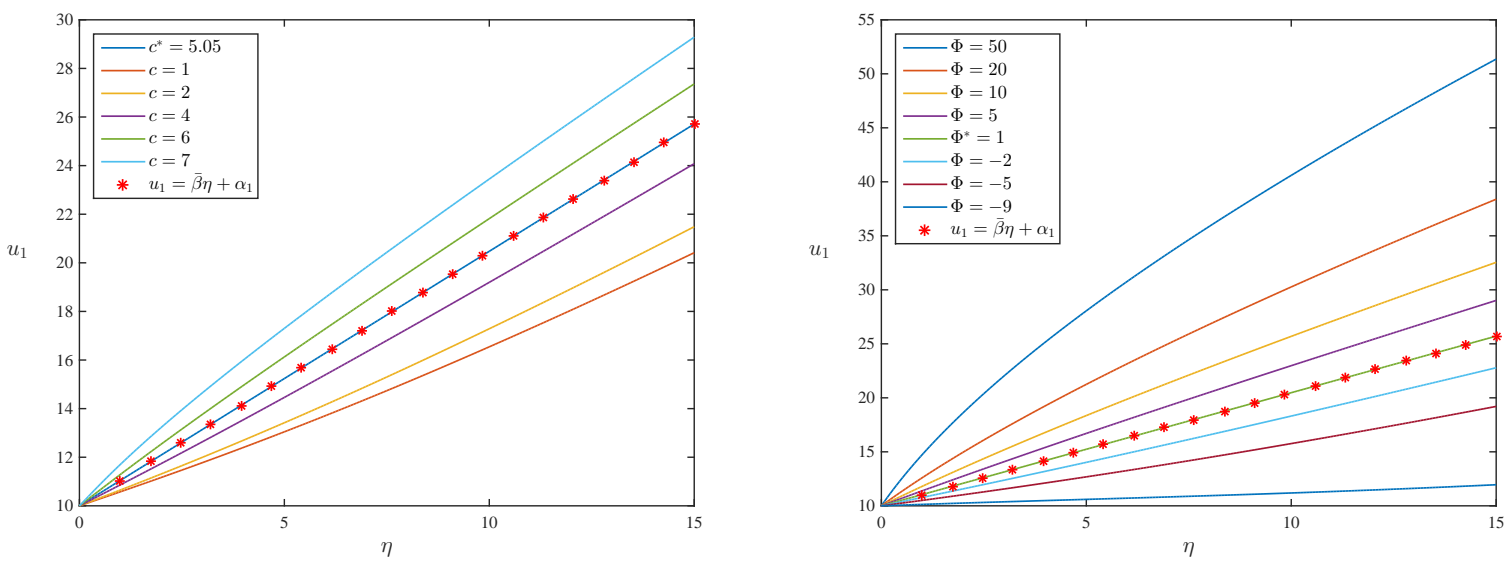

Figure 10: Traveling wave velocity profiles $u_{1}$ against $\eta$. The starred values correspond to the cases where linear behavior is observed. In the left graph we take different values of $c$, for $\Phi_{1}=-3, \Phi_{2}=-7$ and $u_{1}(0)=10, u_{2}(0)=7.61$. In the right graph we take different values of $\Phi$, for $c=-1.0033, u_{1}(0)=10, u_{2}(0)=9.078$

for $v_{1}(x, t)$. Here the parameter $\alpha=2 E^{3} p_{12}>0$.

Consider the initial data

$$
v_{1}(x, 0)=a x+b .
$$

Assuming a solution form

$$
v_{1}(x, t)=(a x+b) g(t),
$$

where $g(0)=1$, we find that $g(t)$ must satisfy the equation

$$
\frac{d g}{d t}+a g^{2}=\alpha g
$$

The solution to this equation is

$$
g(t)=\frac{\alpha}{a+(\alpha-a) e^{-\alpha t}} .
$$

We therefore obtain the exact solution

$$
v_{1}(x, t)=\frac{\alpha(a x+b)}{a+(\alpha-a) e^{-\alpha t}}
$$

to (75). This solution initially has slope $a$ at $t=0$ and gradually approaches a steady state solution with slope $\alpha$ as $t \rightarrow \infty$. Hence, the dynamics are very regular.

Let us now turn our attention to the inviscid Burger's equation

$$
\frac{\partial W}{\partial t}+\frac{1}{2} \frac{\partial}{\partial x}\left(W^{2}\right)=0
$$

with the initial condition

$$
W(x, 0)=a x+b .
$$

Following the same method used above, we find the exact solution

$$
W(x, t)=\frac{a x+b}{a t+1} .
$$

Unlike the regular solution (80), this solution is in general irregular, and in particular develops a singularity at $t=$ $-1 / a$. If the initial slope is negative $(a<0)$, then this means that the solution will blow-up at finite time $t_{\text {blow-up }}=1 /|a|$.

What this comparison illustrates is that solutions to the system (22)-(23) are likely to be more regular or stable than those of the inviscid Burger's equation. This may help to explain why shocks or other such behaviors which 
might lead to turbulence are not found in the gas laser model. Physically, the right hand side of the system (22)-(23) tends to act as a damping field, akin to a kind of discrete diffusion. Indeed, the physical rationalization for the terms on the right hand side of (22)-(23) is that they should encode a kind of discrete diffusion between the two gas states considered.

Returning to the system of two equations, we more generally find that numerical simulations such as that provided in Figure 5 show solutions which tend to regular steady state solutions. In contrast, the same initial conditions used in obtaining Figure 5 will result in shock formation in the inviscid Burger's equation. Hence, even for cases in which $p_{12} \neq p_{21}$, we see very regular dynamics emerge from the numerical simulation of the non-dimensional gas laser model.

\section{Discussion}

We have gone through the derivation of a model for the velocity profiles of the ground and excited state particles of a closed two-level atom gas laser, and obtained a non-dimensional form of the model which is amenable to mathematical analysis. The mathematical analysis showed that there is an unstable uniform zero steady state and a locally stable non-uniform steady state which is linear in the space variable. The slopes of the linear steady state grow algebraically with the energy $E$ for fixed values of $p_{i j}$, and they are heavily influenced by the sum $p_{12}+p_{21}$. The slopes also grow linearly for increasing values $p_{12}=p_{21}$ for fixed values of the energy $E$. The numerical simulations indicate that any initially abnormal behavior is smoothed out over time and the velocity profiles reach the linear steady state, even though a variety of initial conditions are used. Although we can only show that the linear steady state is locally stable using the mathematical analysis, the numerical results suggest that it is likely globally stable.

We then examined a traveling wave solution for the velocity profiles of the ground and excited state. The stability analysis showed that the uniform zero steady state is unstable, as was also shown for the original PDE system. We then examined conditions for linear solutions to exist and arrived at exactly the same conditions for the slopes of the linear solutions of the traveling waves as the ones we found for the linear steady states of the PDE system. We also found a specific relation between the speed of the traveling wave, the forcing parameters, the slopes of the linear solutions, and the parameters $p_{i j}$ and $\mu_{i j}$, in order to obtain a linear solution. We then carried out the numerical simulations for the traveling wave ODE system and examined how different values of wave speed and forcing parameters alter the behavior of the velocity profiles under the traveling wave assumption. Although the numerical simulation for the original PDE system showed that the velocity profiles approach the linear steady state in any case, we observed that linear solutions for the traveling wave solution exist only under specific conditions on the wave speed. In light of the results we have obtained in previous section on the full PDE, it is likely that the wave speed corresponding to a linear profile is the physically meaningful wave speed that would be found experimentally in the two-level gas laser.

As shown in Section 7, the terms on the right hand side of our non-dimensional model, which describe the transition of the two-levels between energy states, act to dampen the solutions, resulting in more regular solutions that would be expected from, say, the inviscid Burger's equation. More generally, we see this in all of the analytical solutions obtained, as well as in all of the numerical simulations. While numerical simulations are not exhaustive, combined with the stability of the steady states, we find it likely that all solutions to the non-dimensional two-state gas laser model remain regular and single-valued. This is not to say that turbulence is impossible in gas laser models, Indeed, for other models related to Burgers' equation, turbulence is certainly possible [19, 20, 21, 22]. However, that solutions to the particular model we study appear regular, with transients dissipating quickly as the solutions tend to a steady state. As steady state turbulence is possible in Burgers' systems [23], the fact that the steady state solutions obtained are very well-behaved and regular is likely a strong indicator that there should be no turbulence in the particular model we consider.

Note that the model (12)-(13) we use is only one possible model, and the results suggest that the model is likely only valid for very regular dynamics in real-world experiments. For other regimes, such as if turbulence is found in real gas lasers experimentally, a different model - perhaps one which takes into account statistical quantities which are integrated out of the present model - may be required. Or perhaps even a different modeling approach would be required. Furthermore, there are several definitions of turbulence, and our focus was to rule out the multi-valued velocity fields claimed in [6], which is one route toward turbulence. Of course, this is one specific model, and there may be turbulence in more realistic models for the gas laser system. As such, there may still be turbulence in a generic gas laser system, however the model needed would likely need to be more involved. In particular, the transition terms 
between states appear to suppress shock solutions that would be found in the inviscid Burger's equation (and which would possibly lead to a breakdown of solutions leading to turbulence), so the inclusion of these interactions between states appears to be the regularizing feature. This is important physically, as it suggests that the behavior under the model we study is more regular and less likely to give way to blow-up, shocks, or lead to the onset of turbulence. However, as the model we study had several underlying assumptions, one may get similar or different dynamics. Therefore, if one was to model the interactions between states differently, or perhaps via nonlinear rather than linear terms, then perhaps more exciting non-equilibrium dynamics would be possible. For example, perhaps one should consider a more general model

$$
\begin{aligned}
& \frac{\partial v_{1}}{\partial t}+\frac{1}{2} \frac{\partial}{\partial x}\left(v_{1}^{2}\right)=A v_{2}+\mathcal{I}_{1}\left(v_{1}, v_{2}\right)+\phi_{1}(x, t), \\
& \frac{\partial v_{2}}{\partial t}+\frac{1}{2} \frac{\partial}{\partial x}\left(v_{2}^{2}\right)=A v_{1}+\mathcal{I}_{2}\left(v_{1}, v_{2}\right)+\phi_{2}(x, t),
\end{aligned}
$$

where $\mathcal{I}_{j}(j=1,2)$ are more general nonlinear interaction terms which arise from different "course-graining" approaches. One interesting area of future work would be to derive a form of this model for physically meaningful yet nonlinear $\mathcal{I}_{j}$, perhaps obtaining a model involving certain nonlinear reaction kinetics which arise in problems of chemical mixtures (with one state serving as activator and the other as inhibitor). In that case, one might find a behavior akin to the Turing instability found in reaction-diffusion models. Here the difference would be that, instead of diffusion, one would have a kind of nonlinear convective transport. Such dynamics would be quite interesting to study, particularly if something like the Turing instability results, as it could provide a route to spatio-temporal chaos and hence turbulence.

It is also possible to consider an $n$-state generalization to the model we study. Preliminary results suggest that the three-state model also exhibits regular dynamics. However, there may be some pathological examples of parameter regimes or initial conditions for which an $n$-state generalization of (12)-(13) could give shocks or other dynamics which may lead to turbulence. As such, the $n$-state generalization would be an interesting topic for future work.

\section{References}

[1] O. Svelto, Principles of lasers. Springer (1998), Chapter 10.

[2] A. Javan, W. R. Bennett, and D. R. Herriott, Population Inversion and Continuous Optical Maser Oscillation in a Gas Discharge Containing a He-Ne Mixture, Physical Review Letters 63 (1961) 106-110.

[3] A. D. White, Recollections of the First Continuous Visible Laser, Optics and Photonics News 22 (2011) 34-39.

[4] C. S. Willet, An Introduction to Gas Lasers. Pergamon Press (1974), pp. 407-411.

[5] F. T. Arecchi, R. Meucci, G. Puccioni, and J. Tredicce, Experimental evidence of subharmonic bifurcations, multistability, and turbulence in a Q-switched gas laser, Physical Review Letters 49(17) (1982) 1217.

[6] M. P. Solon, P. Esguerra, and A. Muriel, Turbulence in a gas laser, Physica A 388(20) (2009) 4361-4363.

[7] S. A. Orszag, Analytical theories of turbulence, Journal of Fluid Mechanics 41 (1970) 363-386.

[8] F. Gao, An analytical solution for the scalar probability density function in homogeneous turbulence, Physics of Fluids A: Fluid Dynamics 3(4) (1991) 511-513.

[9] B. Weigand, An extract analytical solution for the extended turbulent Graetz problem with Dirichlet wall boundary conditions for pipe and channel flows, International Journal of Heat and Mass Transfer 39(8) (1996) 1625-1637.

[10] J. C. Maxwell, On the dynamical theory of gases, Philosophical transactions of the Royal Society of London 157 (1967) 49-88.

[11] R. C. Hilborn, Einstein coefficients, cross sections, f values, dipole moments, and all that, American Journal of Physics 50(11) (1982) 982-986.

[12] J. M. Burgers, A mathematical model illustrating the theory of turbulence, Advances in Applied Mechanics 1 (1948) 171-199.

[13] A. Quarteroni, R. Sacco, and F. Saleri, Numerical Mathematics, (Vol. 37) Springer Science \& Business Media (2010).

[14] M. Wang, X. Li, and J. Zhang, The (G'/G)-expansion method and travelling wave solutions of nonlinear evolution equations in mathematical physics, Physics Letters A 372 (2008) 417-423.

[15] A.M. Wazwaz, The tanh method for traveling wave solutions of nonlinear equations, Applied Mathematics and Computation 154 (2004) 713-723.

[16] E.J. Parkes and B. R. Duffy, An automated tanh-function method for finding solitary wave solutions to non-linear evolution equations, Computer Physics Communications 98 (1996) 288-300.

[17] W. Malfliet, Solitary wave solutions of nonlinear wave equations, American Journal of Physics 60 (1992) 650-654.

[18] P. D. Lax, Integrals of nonlinear equations of evolution and solitary waves, Communications on Pure and Applied Mathematics 21(5) (1968) 467-490.

[19] E. Balkovsky, G. Falkovich, I. Kolokolov, and V. Lebedev, Intermittency of Burgers’ turbulence, Physical Review Letters 78(8) (1997) 1452.

[20] J. P. Bouchaud, M. Mézard, and G. Parisi, Scaling and intermittency in Burgers turbulence, Physical Review E 52(4) (1995) 3656.

[21] M. Lässig, Dynamical anomalies and intermittency in burgers turbulence, Physical Review Letters 84 (2000) 2618. 
[22] R. Iturriaga and K. Khanin, Burgers turbulence and random Lagrangian systems, Communications in Mathematical Physics 232 (2003) $377-428$

[23] T. Gotoh and R. H. Kraichnan, Steady-state Burgers turbulence with large-scale forcing, Physics of Fluids 10 (1998) $2859-2866$. 